\title{
Magnetization Modeling of Twisted Superconducting Filaments
}

\author{
T. Satiramatekul, F. Bouillault, A. Devred, and D. Leroy
}

\begin{abstract}
This paper presents a new Finite Element numerical method to analyse the coupling between twisted filaments in a superconducting multifilament composite wire. To avoid the large number of elements required by a 3D code, the proposed method makes use of the energy balance principle in a $2 \mathrm{D}$ code. The relationship between superconductor critical current density and local magnetic flux density is implemented in the program for the Bean and modified Kim models. The modeled wire is made up of six filaments twisted together and embedded in a low-resistivity matrix. Computations of magnetization cycle and of the electric field pattern have been performed for various twist pitch values in the case of a pure copper matrix. The results confirm that the maximum magnetization depends on the matrix conductivity, the superconductor critical current density, the applied field frequency, and the filament twist pitch. The simulations also lead to a practical criterion for wire design that can be used to assess whether or not the filaments are coupled.
\end{abstract}

Index Terms-Coupling currents, energy balance principle, magnetization, superconducting multifilament composite wire, twist.

\section{INTRODUCTION}

$\mathbf{T}$ HE numerical simulation reported here allows us to define the coupling-decoupling phenomena between superconducting filaments. We have shown [1] that the magnetization obtained in the case of uncoupled superconducting filaments is smaller than the one obtained for coupled superconducting filaments.

In practical multifilament composite wires, the superconducting filaments are embedded in a low-resistivity matrix that is much less conducting than the superconductor, for example, OFHC copper. Then, there are currents circulating between the filaments via the conducting matrix, which result in a filament coupling. This coupling can be greatly reduced by twisting the filaments together [2]. The shorter the twist pitch, the less coupled the filaments. An electromagnetic theory of this problem is presented in [3].

This paper deals with the electromagnetic coupling under external field, under self field the coupling is different and the twisting plays no part. We use a method based on the principle of energy balance to solve the problem of twisted filaments. We

Manuscript received August 19, 2006.

T. Satiramatekul is with the Faculty of Engineering, Kamphaengsaen, Kasetsart University, Kamphaengsaen, Nakhon Pathom 73140, Thailand (e-mail: thitipong.s@ku.ac.th).

F. Bouillault is with LGEP, CNRS UMR 8507, SUPELEC, Plateau de Moulon, 91192 Gif-sur-Yvette Cedex, France.

A. Devred is with CEA, DSM/DAPNIA/SACM, 91191 Gif-Sur-Yvette Cedex, France, and CERN, AT-MAS, CH-1211, Geneva 23, Switzerland.

D. Leroy is with CERN, AT-MAS, CH-1211, Geneva 23, Switzerland.

Digital Object Identifier 10.1109/TASC.2007.899414

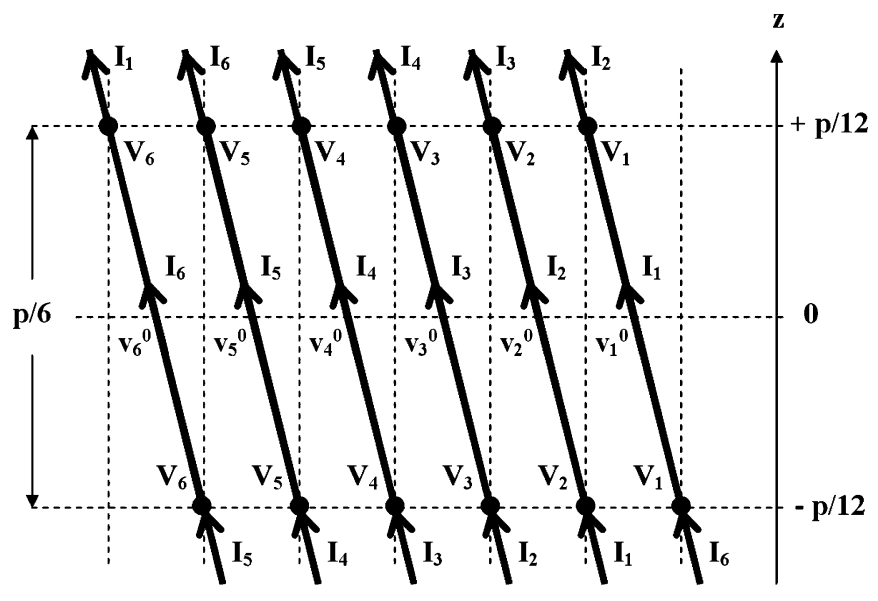

Fig. 1. 2D representation of six-filament wire over one sixth of a twist pitch.

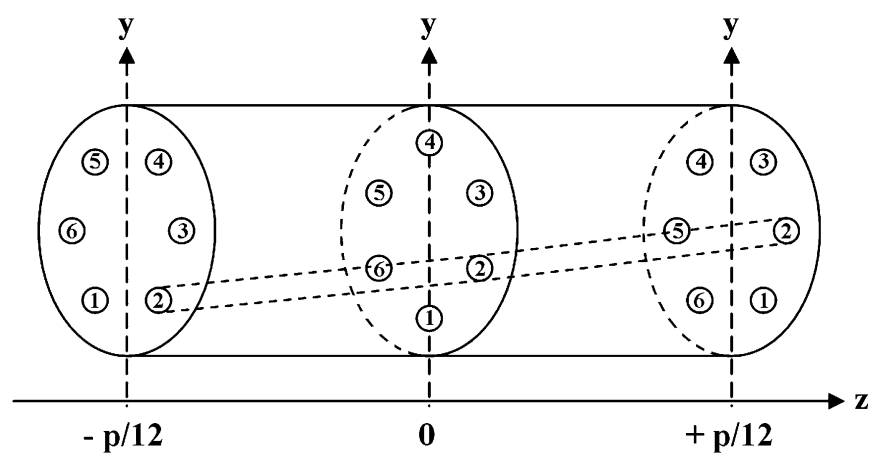

Fig. 2. Position of six filaments in the planes $z=0$ and $z= \pm p / 12$.

also study how the dependence of the current density on the magnetic flux density affects the magnetization of twisted superconducting filaments.

\section{TWISTED WIRE MODEL}

In order to understand the influence of the twisting on the magnetization, we start by considering a composite wire made up of six superconducting filaments, twisted together and embedded in a low-resistivity matrix.

Let us consider the 2D representation given in Fig. 1 of the currents and voltages along a sixth of the filament twist pitch, $p$, as well as the positions of the six filaments in the planes $z=0$ and $z= \pm p / 12$ illustrated in Fig. 2. Given the problem geometry, we assume that the electrical potential, $V_{\mathrm{i}}$, along filament $i$, is of the form

$$
V_{\mathrm{i}}(z)=V_{0} \cos \left[\frac{2 \pi}{p} z+(i-1) \frac{2 \pi}{6}\right]
$$

where $V_{0}$ is a reference potential. 
In addition, for the currents, we assume that

$$
\begin{aligned}
I_{k+1}(z+p / 6) & =I_{k}(z) \quad \text { for } k, 1 \leq k \leq 5 \\
I_{1}(z+p / 6) & =I_{6}(z) .
\end{aligned}
$$

In order to avoid a three-dimensional modeling, we divide the wire into elementary sections of $(p / 6)$ length. Given the weak slope of the filaments, we assume that, in each section, the problem can be treated as two-dimensional and that the $I_{k}$ currents are independent of $z$. Then, the difference of the currents in the filaments for two successive sections is equal to the current circulating in a section of matrix of $(p / 6)$ length subjected to the adequate potentials.

By using the principle of energy balance [4] in the plane $z=$ $\pm p / 12$ in Fig. 1, we derive

$$
\sum_{i=1}^{5} V_{\mathrm{i}}\left(I_{\mathrm{i}+1}-I_{\mathrm{i}}\right)+V_{6}\left(I_{1}-I_{6}\right)=P
$$

where $P$ is the power dissipated in a section of conducting matrix. In this case, we simply have

$$
P=\lambda_{0} \sigma V_{0}^{2} p / 6
$$

and

$$
\lambda_{0}=\int_{\Omega}[\operatorname{grad}(u)]^{2} d \omega
$$

where $\sigma$ is the electrical conductivity of the matrix and $\lambda_{0}$ is a dimensionless parameter obtained by solving the 2D harmonic Laplace equation, which in our formulation corresponds to: $\Delta u=0$. The equation is solved by the finite element method with the boundary conditions

$$
\left.\begin{array}{l}
u_{01}=u_{06}=+\sqrt{3} / 2 \\
u_{02}=u_{05}=0 \\
u_{03}=u_{04}=-\sqrt{3} / 2
\end{array}\right\} .
$$

By substituting the voltages in (4), we obtain get

$$
\sqrt{3} / 2 \times\left(I_{2}+I_{3}-I_{5}-I_{6}\right)=\lambda_{0} \sigma V_{0} p / 6
$$

Equation (8) can be re-written in the following matrix form

$$
[C]^{t} I=-\lambda_{0} \sigma p^{2} v^{0} / 36
$$

where

$$
\begin{aligned}
v^{0} & =-V_{0} / d \approx-V_{0} /(p / 6) \\
{[C] } & =\left[\begin{array}{llllll}
0 & \sqrt{3} / 2 & \sqrt{3} / 2 & 0 & -\sqrt{3} / 2 & -\sqrt{3} / 2
\end{array}\right]^{t}
\end{aligned}
$$

where $d$ is defined in Fig. 3 and $I$ is the current vector in the filaments at $z=0$. The currents can be derived from [1]

$$
I^{n}=\mu_{0}^{-1} \times\left(\left[A_{e v 0}\right]^{t} E+\left[A_{v 0}\right] E_{0}\right)^{n} \times \Delta t+I^{n-1}
$$

with

$$
E_{0}=[C] v^{0}
$$

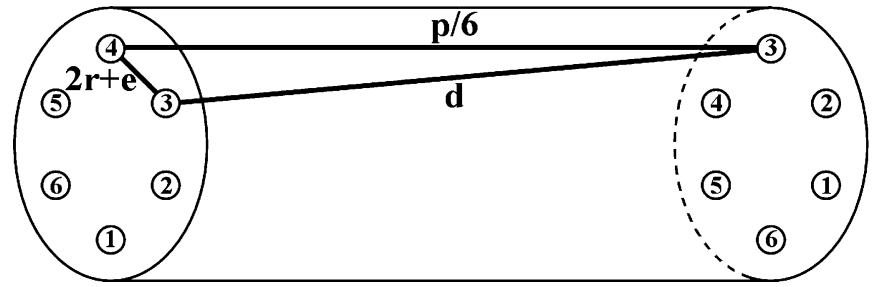

Fig. 3. Definition of the distance $d$ for one sixth of a twist pitch.

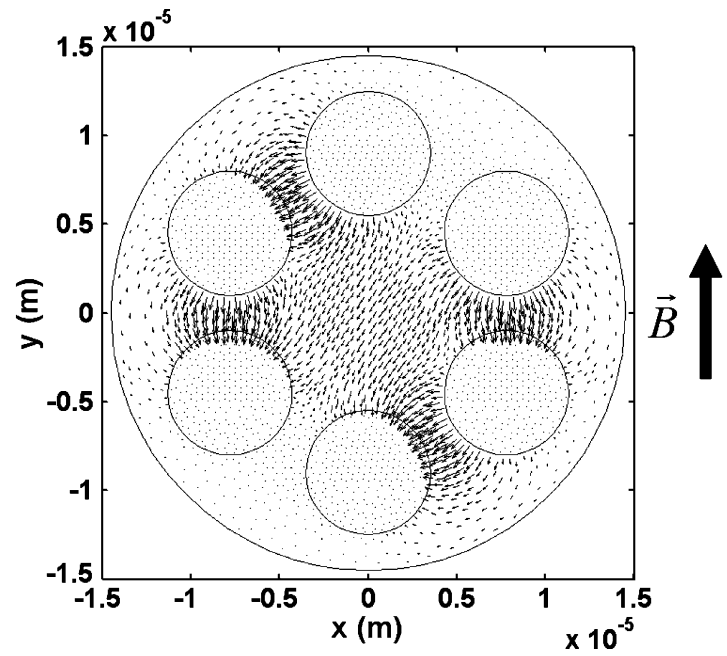

Fig. 4. Current density distribution in conducting matrix (in $\mathrm{A} / \mathrm{m}^{2}$ ).

where $\mu_{0}$ is the magnetic permeability of vacuum, $E$ is the electrical field vector, and $\left[A_{e v 0}\right]$ and $\left[A_{v 0}\right]$ are matrices whose dimensionless coefficients take into account the wire geometry.

The matrix system resulting from the discretization of Maxwell's equations in the case of the twisted filaments is [1]

$$
\begin{aligned}
\mu_{0}[M] \partial_{t} J+\left[A_{e 0}\right] E+\left[A_{e v 0}\right][C] v^{0} & =\mu_{0} F \\
-\left(\frac{\mu_{0} \lambda_{0} \sigma p^{2}}{36 \Delta t}+[C]^{t}\left[A_{v 0}\right][C]\right) v^{0}= & {[C]^{t}\left[A_{e v 0}\right]^{t} E } \\
& +\frac{\mu_{0}}{\Delta t}[C]^{t} I^{n-1}
\end{aligned}
$$

where $J$ is the current density vector, $F$ is the source vector, $[M]$ is the mass matrix, and $\left[A_{e 0}\right]$ is a dimensionless matrix.

On the left side of (15), we find that, if the first term is very small compared to the second term, we are in the case of uncoupled filaments. Conversely, if the first term is very large compared to the second term, we are in the case of coupled filaments [1].

In addition, the current density in the conducting matrix can be derived from

$$
j=\sigma V_{0} \operatorname{grad}(u) \text {. }
$$

Fig. 4 shows the simulation results in the plane $z=+p / 12$. In order to compute the total magnetization of a multifilament wire, let us consider the 2D drawing of the currents in Fig. 5. We find that there are two contributions: one from the currents in the filaments, and one from the currents in the conducting matrix. 


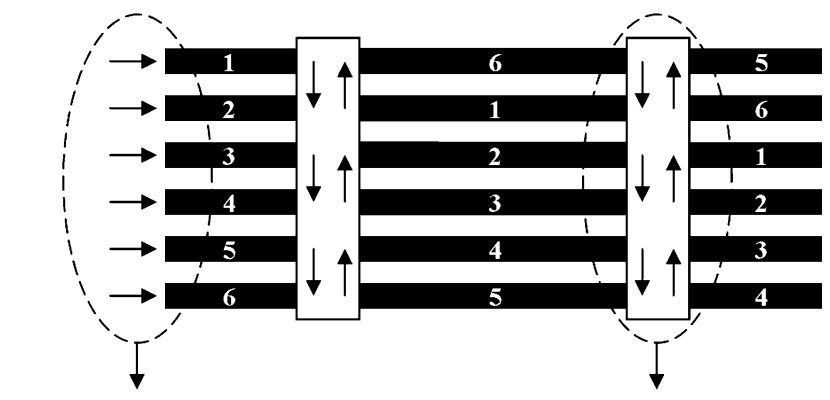

Currents in the filaments

Fig. 5. 2D Drawing of currents in wire with twisted filaments.

The total magnetization per superconductor unit volume is

$$
\begin{aligned}
\vec{M} & =\left(\int_{V} \vec{r} \times\left(\vec{j}_{\text {filaments }}+\vec{j}_{\text {matrix }}\right) d v\right) /(L \times S) \\
\vec{M} & =\vec{M}_{\text {filaments }}+\vec{M}_{\text {matrix }}
\end{aligned}
$$

where $L$ is the wire length and $S$ is the superconductor crosssectional area, and $\vec{M}_{\text {filaments }}$ and $\vec{M}_{\text {matrix }}$ are the magnetizations due to the currents circulating in the superconducting filaments and in the conducting matrix.

In our case, it can be shown that

$$
\vec{M}_{\text {matrix }}=\overrightarrow{0}
$$

which yields

$$
\vec{M}=\vec{M}_{\text {filaments }}=\int_{S_{\text {filaments }}}\left(y j \vec{u}_{x}-x j \vec{u}_{y}\right) d s / S
$$

where $j$ depends on the filament twist pitch.

\section{Simulation Results AND Discussion}

The finite elements code applied for superconductors was developed by LGEP. Our work is based on this code. The method consists in using a model with two slopes instead of Bean's model [5]. But for the superconductors at high critical temperature, the real characteristic $E(J)$ differs rather clearly from that of Bean's model, a more realistic model can be obtained by a progressive function [6]. We modeled a wire made up of six superconducting filaments twisted together and embedded in an OFHC copper matrix $\left(\sigma=8.82 \times 10^{9} \mathrm{~S} / \mathrm{m}\right.$ at $\left.4.2 \mathrm{~K}\right)$. A time-varying magnetic flux density was applied vertically with a maximum amplitude of $1 \mathrm{~T}$ and a frequency of $1 \mathrm{~Hz}$. Initially, the simulations were made according to Bean's model and assuming a constant critical current density of $7 \times 10^{10} \mathrm{~A} / \mathrm{m}^{2}$. In order to study the influence of twisting on the coupling between filaments and on the magnetization, we varied the filament twist pitch of the filaments, $p$, from $0.03 \mathrm{~mm}$ to $300 \mathrm{~mm}$. Fig. 6 illustrates the current density distributions in the six filaments in the plane $z=0$ for two extreme twist pitch values. In Fig. (6a) $(p=300 \mathrm{~mm})$, we can see the case of perfectly coupled filaments, while in Fig. (6b) $(p=0.03 \mathrm{~mm})$, we can

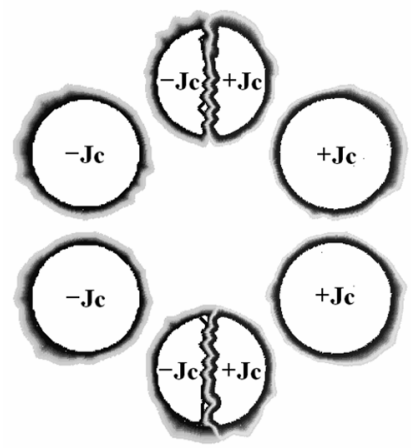

(a)

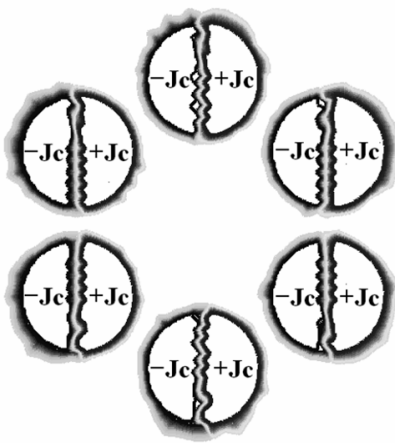

(b)
Fig. 6. Current density distributions at $t=T / 4$ for (a) $p=300 \mathrm{~mm}$ and (b) $p=0.03 \mathrm{~mm}$ (using Bean's model).

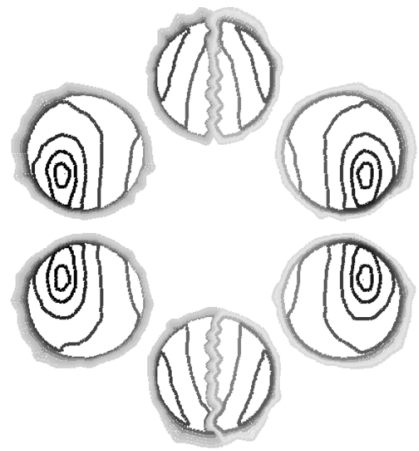

(a)

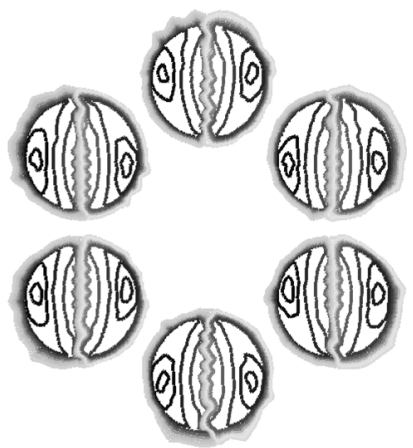

(b)
Fig. 7. Current density distributions at $t=T$ for (a) $p=300 \mathrm{~mm}$ and (b) $p=0.03 \mathrm{~mm}$ (using Kim's model).

see the case of perfectly decoupled filaments. When decoupled, the filaments carry their own go and return currents, whereas coupled filaments behave like one monofilament conductor. For intermediate $p$ values, we can observe cases of partial filament coupling.

In order to take into account the dependence of the critical current density on the magnetic flux density, a second set of simulations were carried out using Kim's model, with $J_{c}(B)=$ $J_{c}(0)\left(1+B / B_{0}\right)^{-1}$ with $J_{c}(0)=7 \times 10^{-10} \mathrm{~A} / \mathrm{m}^{2}$ and $B_{0}=$ $0.1 \mathrm{~T}$ [1]. The modeled domain, the matrix conductivity value and the applied magnetic flux densities are the same ones as those used previously. Fig. 7 shows the current density distributions in the six filaments in the plane $z=0$ for the same two extreme cases, where again observe perfect coupling and decoupling [2].

Figs. 8 and 9 compare the magnetization cycles obtained using Bean's and Kim's model for three different twist pitch values. We can clearly see the deformation of the cycle in the case of partially coupled filaments compared to the extreme cases of perfect coupling and uncoupling. In the case of Kim's model, the deformation is more important at high magnetic flux densities than at low magnetic flux densities. This deformation is due to the current density distribution in the filaments [4].

From the magnetization cycles, we can deduce the maximum magnetization value. According to our computations, we have 


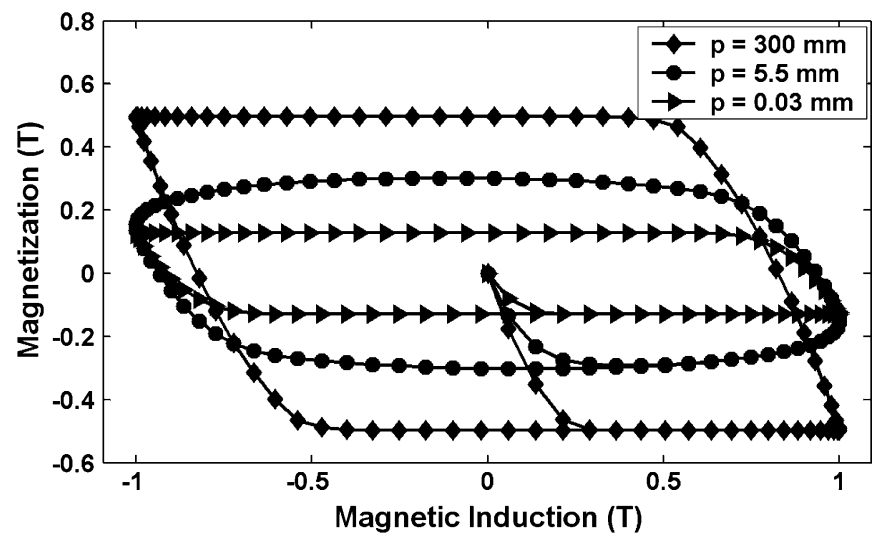

Fig. 8. Magnetization cycles obtained from Bean's model.

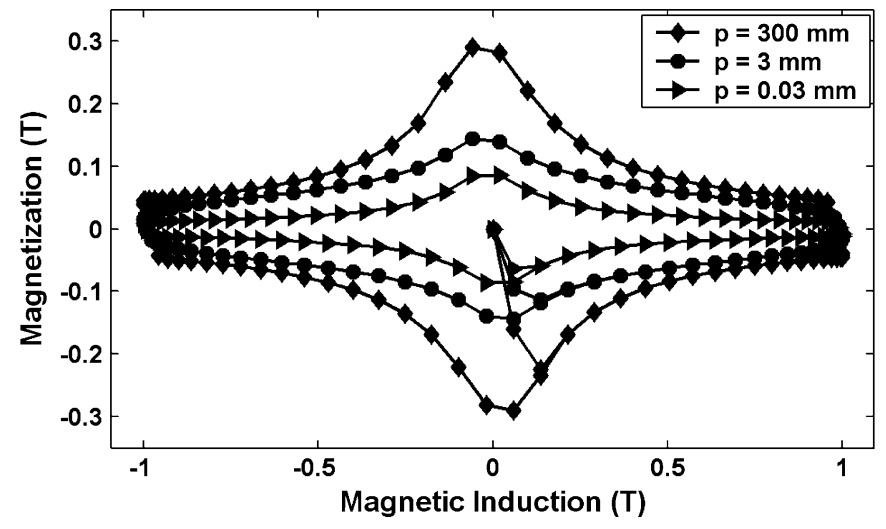

Fig. 9. Magnetization cycles obtained from Kim's model.

confirmation that this value depends on the electrical conductivity of the conducting matrix, on the critical current density, on the frequency of the applied magnetic induction, and on the twist pitch of the filaments. From (15), it appears that a good intrinsic parameter to characterize the effect of twisting for a given critical current density is: $\mu_{0} \sigma p^{2} f$ or: $p / \delta$, where $\delta$ is the skin effect in the conducting matrix. Fig. 10 shows that, for Bean's model with $J_{c}(0)=7 \times 10^{-10} \mathrm{~A} / \mathrm{m}^{2}$, the filaments are coupled together when $p / \delta$ is higher than 5.60. On the other hand, if this value is lower than 0.056 , the filaments can be considered as decoupled. Then, in practice, if copper is used for the conducting matrix, it is necessary that the filament twist pitch be lower than $1 \mathrm{~mm}$ to unsure that so that the superconducting filaments are decoupled. When relying on Kim's model, the filaments are coupled if the $p / \delta$ value is higher than 1.90 , and decoupled if the $p / \delta$ value is lower than 0.056 . Note that this lower limit is the same as the one obtained with Bean's model, and that in our computation where $J_{\mathrm{c}}(0)=J_{\mathrm{c}}=7 \times 10^{-10} \mathrm{~A} / \mathrm{m}^{2}$, we

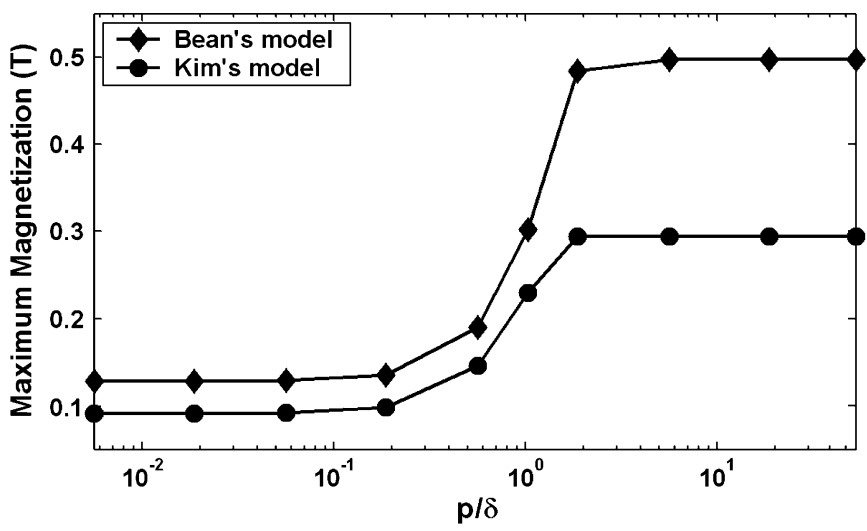

Fig. 10. Maximum magnetization versus $p / \delta$ ratio.

find that the maximum magnetization obtained by Kim's model is always smaller than that obtained by Bean's model. The difference between two values is more important in the case of coupled filaments than for uncoupled filaments.

\section{CONCLUSION}

We studied the effects of superconducting filament twisting in composite wires. We proposed a method based on the principle of energy balance to solve this problem in 2D. Simulations were carried out using Bean's model and Kim's model. In both cases, we could express the maximum magnetization as a function of a reduced parameter directly proportional to the filament twist pitch. The value of this parameter enables to assess the where or not the superconducting filaments are coupled together through the low-resistivity matrix of the wire.

\section{REFERENCES}

[1] T. Satiramatekul and F. Bouillault, "Magnetization of coupled and noncoupled superconducting filaments with dependence of current density on applied field," IEEE Trans. Magn., vol. 41, pp. 3751-3753, Oct. 2005.

[2] M. N. Wilson, Superconducting Magnets. Oxford: Oxford University Press, 1983.

[3] W. J. Carr, Jr., "Electromagnetic theory for filamentary superconductors," Phys. Rev. B, vol. 11, pp. 1547-1554, Feb. 1975.

[4] T. Satiramatekul, F. Bouillault, and L. Santandrea, "Modeling of magnetization curves of partially coupled superconducting filaments with dependence of current density according to applied magnetic field," IEEE Trans. Magn., vol. 42, pp. 887-890, Apr. 2006.

[5] M. Maslouh, F. Bouillault, A. Bossavit, and J. C. Verite, "From Bean's model to the H-M characteristic of a superconductor: Some numerical experiments," IEEE Trans. Appl. Supercond., vol. 7, pp. 3797-3800, Sep. 1997.

[6] N. Banno and N. Amemiya, "Numerical analysis of AC loss in high $\mathrm{T}_{\mathrm{C}}$ twisted tape carrying AC transport current in external AC magnetic field. Effect of twisting on loss reduction," IEEE Trans. Appl. Supercond., vol. 9, pp. 2561-2564, June 1999. 